\title{
A PERSUASÃO INCONSCIENTE: UM MODELO CONCEITUAL SOBRE OS EFEITOS DA MULTITAREFA DE MÍDIA SOBRE A FLUÊNCIA DE PROCESSAMENTO DO CONSUMIDOR
}

Objetivo: Direcionar novos estudos de fluência de processamento no contexto relativamente novo da multitarefa de mídia, comportamento que tem se tornado popular nos últimos anos.

Método: Revisão da vasta literatura de fluência de processamento, fluência perceptual e fluência conceitual, incluindo seus diversos efeitos, diferentes contextos comportamentais e as teorias que explicam o fenômeno. Realizou-se também uma breve revisão sobre a recente literatura da multitarefa e multitarefa de mídia.

Originalidade/Relevância: Estímulos fluentes possuem vários efeitos positivos no consumidor em relação a estímulos não fluentes, contudo, todos os estudos de fluência até agora consideraram indivíduos focados em uma atividade durante a exposição ao estímulo, o que não representa grande parte do comportamento habitual dos indivíduos que costumam se engajar em mais de uma atividade ao mesmo tempo, principalmente ao consumir uma mídia. Este trabalho destaca este gap e fornece direções para futuros trabalhos.

Resultados: A partir da revisão da literatura, este artigo oferece proposições de estudo e um modelo conceitual que podem orientar futuras pesquisas sobre fluência de processamento (perceptual e conceitual) em situações em que o consumidor desempenha mais de uma atividade ao mesmo tempo.

Contribuições teóricas / metodológicas: Este artigo contribui para a melhor compreensão dos efeitos de exposições a estímulos a partir do melhor entendimento dos efeitos da fluência perceptual e conceitual e como esses efeitos podem ser afetados pelo comportamento multitarefa de mídia.

Palavras-chave: Fluência de Processamento. Fluência Perceptual. Fluência Conceitual. Multitarefa de Mídia. Comportamento do Consumidor.

\section{THE UNCONSCIOUS PERSUASION: A CONCEPTUAL MODEL ABOUT THE MEDIA MULTITASKING EFFECTS ON CONSUMER'S PROCESSING FLUENCY}

Objective: Provide directions to new processing fluency studies in the relatively new context of the multitasking media, a behavior that has become popular in recent years.

Method: Review of the robust literature of processing fluency, perceptual fluency and conceptual fluency, including its various effects, different behavioral contexts and theories that explain the phenomenon. In addition, it was made a brief review about the recent literature of multitasking and media multitasking was.

Originality / Relevance: Fluent stimuli have several positive effects on the consumer in comparison to non-fluent stimuli; however, all fluency studies so far have considered individuals focused on a single activity during stimulus exposure, which does not represent the realistic behavior during exposures, since individuals usually engage in more than one activity at a time, especially when consuming media. This article highlights this gap and provides directions for future work.

Results: From the literature review, this article offers study propositions and a conceptual model that can guide future research on processing fluency (perceptual and conceptual) in situations where the consumer performs more than one activity at the same time.

Theoretical / methodological contributions: This article contributes to a better understanding of the effects of stimulus exposures through a better understanding of the effects of perceptual and conceptual fluency and how these effects can be affected by multitasking media behavior.

Keywords: Processing Fluency. Perceptual Fluency. Conceptual Fluency. Media Multitasking. Onsumer Behavior.

Sylvio Ribeiro ${ }^{1}$ Daniel Max de Sousa Oliveira ${ }^{2}$ Martin de La Martinière Petroll ${ }^{3}$

\footnotetext{
${ }^{1}$ Doutorando em Administração pelo Programa de Pós-Graduação da Universidade Federal do Rio Grande do Sul - UFRGS. Rio Grande do Sul, Brasil. E-mail: sylvio.ros@gmail.com

2 Mestre em Administração pela Universidade Federal de Santa Catarina - UFSC. Santa Catarina, Brasil. E-mail: danielmax2011@gmail.com

${ }^{3}$ Doutor em Administração pela Universidade Federal do Paraná - UFPR. Professor da Universidade Federal de Santa Catarina - UFSC. Santa Catarina, Brasil. E-mail: martin.petroll@ufsc.br
} 


\section{INTRODUÇÃO}

Ao longo dos anos, a fluência de processamento $^{i}$, que se refere à formação de uma avaliação positiva em relação a um estímulo, em decorrência da facilidade com que ele é processado, tem sido analisada em uma série de contextos diferentes para explicar a tendência dos seres humanos a reagirem positivamente diante de um estímulo neutro com o qual tiveram contato anteriormente. Por exemplo, estudos têm demonstrado que indivíduos consideram afirmações fluentes, isto é, declarações processadas mais rapidamente, como mais verdadeiras (Begg, Anas, \& Farinacci, 1992; McGlone \& Tofighbakhsh, 2000; Reber \& Schwarz, 1999) mais famosas (Jacoby, Woloshyn, \& Kelley, 1989), mais familiares (Monin, 2003; Tanner \& Maeng, 2012; Whittlesea, 1993), mais apreciáveis (Reber, Winkielman, \& Schwarz, 1998; Zajonc, 1968) e oriundas de fontes com maior credibilidade em comparação a afirmações de baixa fluência (Oppenheimer, 2006).

Em estudos empíricos na área de marketing, a fluência já foi aplicada na avaliação de marcas em exposições incidentais (Janiszewski, 1993; Shapiro \& Nielsen, 2013), na complexidade e repetição de marcas (Janiszewski \& Meyvis, 2001; Nordhielm, 2002), na posição da imagem em anúncios (Chae \& Hoegg, 2013) e na congruência entre texto e imagem (van Rompay, de Vries, \& van Venrooij, 2010). De maneira geral, esses estudos demonstram que a exposição a elementos já vistos, ainda que de forma inconsciente, estimula a formação de reações mais positivas em comparação a elementos inéditos. Isso explica o fato de um produto poder ser escolhido não pelo que oferece ou o conhecimento do consumidor sobre ele, mas pela facilidade com que um produto exposto é processado pela mente do consumidor (Nedungadi, 1990). De certo modo, a fluência pode ser considerada, praticamente, a única fonte de preferência não baseada em atributos ou benefícios (Janiszewski, Kuo, \& Tavassoli, 2013; Schwarz, 2004).

Desse modo, a fluência pode ajudar as empresas a criarem mensagens mais eficazes (Shapiro \& Nielsen, 2013) ao facilitar a identificação, reconhecimento e processamento do conteúdo de marketing (Lee \& Labroo, 2004), aumentando, assim, a confiança na escolha em uma situação de compra por impulso ou de baixa elaboração (Tsai \& McGill, 2011), além de melhorar a avaliação da marca ou produto (Nordhielm, 2002) e aumentar a preferência por eles (Janiszewski \& Meyvis, 2001; Krishnan \& Shapiro, 1996).

Apesar da relevância da fluência para o marketing e comportamento do consumidor, estudos sobre a fluência no meio digital, e que considerem padrões comportamentais típicos desse meio, como utilizar mais de uma mídia simultaneamente (multitarefa de mídia) ainda não foram realizados. Grande parte dos estudos sobre fluência foi publicada antes da massificação das redes sociais e da internet móvel e, portanto, utilizaram metodologias que objetivavam a representação do meio físico, como anúncios impressos (Janiszewski, 1993; Lee \& Aaker, 2004; Shapiro, 1999; Shapiro \& Nielsen, 2013), situações de escolhas de produtos (Novemsky, Dhar, Schwarz, \& Simonson, 2007) e cupons de descontos impressos (Kramer \& Kim, 2007). Dentre os poucos artigos que analisaram a fluência no contexto digital estão o estudo da congruência entre a imagem e o texto em sites de serviços (van Rompay et al., 2010), a análise de conteúdo de tweets demonstrando que o uso de hashtags, links e menções aumentam a disfluência (Pancer \& Poole, 2016), isto é dificultam o processamento de informações, do mesmo modo a complexidade visual em lojas virtuais mobile (Sohn, 2017).

Diante da importância da fluência para o marketing e a baixa quantidade de estudos aplicados ao contexto digital e nenhum considerando as diferenças entre o comportamento off-line e on-line, o objetivo deste artigo é fazer uma breve revisão da vasta literatura sobre fluência de processamento ${ }^{\text {ii }}$, conceituando e destacando os estudos na área de marketing e comportamento do consumidor no meio digital. Ao final, foi elaborado um modelo teórico e proposições de pesquisa que possam direcionar os estudos futuros sobre fluência em contextos digitais e tecnológicos.

\section{REFERENCIAL TEÓRICO}

Esta seção apresentará uma pequena revisão da literatura sobre fluência de processamento e, ao final, apresentará o recente fenômeno da multitarefa de mídia. Para a revisão da literatura de fluência de processamento, diversas buscas foram realizadas nas bases de dados EBSCOhost e Scopus com o intuito de obter as palavras-chaves mais relevantes. A partir das buscas iniciais, e da leitura de diversos artigos sobre fluência, chegou-se aos termos mais utilizados: "processing fluency", "perceptual fluency" e "conceptual fluency". Novas buscas foram realizadas com estes termos e combinações com outros termos como "advertising", "ads", "mere exposure" e "media multitasking". As buscas ocorreram em junho de 2016 e abril de 2017. 


\subsection{Fluência de Processamento}

A exposição a um estímulo facilitará o processamento (codificação, compreensão, percepção e recuperação de informações) deste estímulo em um encontro posterior. Esta facilidade é conhecida como fluência de processamento e leva indivíduos a avaliarem mais positivamente estímulos "conhecidos" do que "desconhecidos", mesmo quando os indivíduos não se lembram de terem sido expostos aos estímulos (Janiszewski \& Meyvis, 2001; Reber et al., 1998). A fluência de processamento pode ser de natureza perceptual ou conceitual (Tulving \& Schacter, 1990). Fluência perceptual refere-se à facilidade de processamento de estímulos visuais conhecidos (Jacoby \& Dallas, 1981) já a fluência conceitual diz respeito à facilidade em acessar as estruturas de conhecimento do campo semântico, por exemplo, extrair significados, comparações ou lembranças espontâneas (Tversky \& Kahneman, 1973; Whittlesea, 1993).

A fluência de processamento sugere que empresas devem introduzir novidades com prudência, pois consumidores são mais favoráveis a objetos que estão de acordo com as suas estruturas de conhecimento e a representação mental que eles possuem acerca destes objetos (Kim, Rao, \& Lee, 2009; Lee \& Aaker, 2004), ou seja, possuem uma tendência natural a preferir o que eles já conhecem . A fluência ocorre quando a representação mental que um indivíduo possui de um objeto corresponde com o que é apresentado na realidade. Os efeitos principais, para o estudo do comportamento do consumidor, são avaliações e atitudes mais positivas em relação ao objeto (Chae \& Hoegg, 2013; Seamon et al., 1995). Isso acontece porque, diante de um estímulo, o processamento de informação é infuenciado por sensações subjetivas de facilidade ou dificuldade.

A fluência é uma experiência metacognitiva (Schwarz, 2004), ou seja, vai além da cognição. É uma sensação agradável associada a uma operação cognitiva. Essa associação acontece de forma automática com praticamente qualquer forma de pensamento (Oppenheimer, 2008; Schwarz, 2004). Experiências fluentes geram interpretações involuntárias sobre o que está ocorrendo e que podem influenciar julgamentos, principalmente quando a experiência acontece de forma inesperada em que o indivíduo não encontra uma justificativa clara para explicar seus julgamentos (Whittlesea \& Williams, 2000). O efeito da falsa fama é um bom exemplo disso (Jacoby, Woloshyn, et al., 1989), ele demonstra que pessoas têm mais chances de considerar nomes famosos se já foram expostos a eles anteriormente. Nesse caso, a fluência de nomes não famosos, devido a exposição anterior, é mal atribuída à fama. Os autores do estudo comentam que os indivíduos que suspeitassem que seus julgamentos podiam ser influenciados por um encontro anterior com o nome, estariam menos suscetíveis a considerar qualquer nome famoso.

Aprofundando mais sobre a fluência, Tulving e Schacter (1990) afirmaram que a fluência de processamento pode ser de dois tipos: fluência perceptual (perceptual fluency ${ }^{i i i}$ ) e fluência conceitual (conceptual fluency ${ }^{i v}$ ). A fluência perceptual é gerada a partir de um estímulo visual (influenciados por repetição, clareza, duração e prototipicidade), que leva o indivíduo a formar uma representação mental das características do estímulo de modo a facilitar a codificação e o processamento em situações futuras com o mesmo estímulo visual (Bornstein \& D’Agostino, 1994; Shapiro, 1999). Portanto, a fluência perceptual é a facilidade com que se percebe e identifica as características físicas de um estímulo e é uma consequência direta de exposições anteriores (Jacoby \& Dallas, 1981). Por exemplo, quando a exposição a um estímulo aciona um traço na memória de forma implícita, isto é, sem que o indivíduo tenha consciência, este indivíduo irá atribuir a facilidade de processamento (fluência) à maior familiaridade ou preferência pelo estímulo ou objeto. Reber, Winkielman e Schwarz (1998) verificaram que a fluência perceptual é naturalmente afetiva-positiva, e não neutra, pois eleva a avaliação de beleza e apreciação dos estímulos, ao mesmo tempo que diminui a avaliação de feiura e a depreciação desses objetos.

Já a fluência conceitual é gerada a partir do significado de um estímulo e sua relação com as estruturas de conhecimento do campo semântico (Whittlesea, 1993). Da mesma forma que a fluência perceptual, o indivíduo também registra uma representação mental ao ser exposto a um estímulo, de modo a facilitar o acesso futuro a ele. No entanto, diferentemente da fluência perceptual, não é preciso entrar em contato novamente com o estímulo para que seu efeito seja ativado (Shapiro, 1999). Portanto, a repetição não é um fator crucial para a fluência conceitual como é para a fluência perceptual. Semelhante à afirmação de Tversky e Kahneman (1973), de que as pessoas costumam fazer julgamentos com base na facilidade com que associações vêm à mente, um estímulo que vem à mente com facilidade pode ser considerado conceitualmente fluente (Lee \& Labroo, 2004).

Lembrar de uma determinada marca a caminho da loja e considerar comprá-la é uma ação associada à fluência conceitual. Este tipo de fluência está presente quando estímulos demandam conhecimentos prévios baseados em conceitos ou significados (ao contrário de atributos da fluência perceptual) já estabelecidos na mente do indivíduo. Para Whittlesea (1993), a fluência conceitual afeta 
qualquer julgamento que envolva processos baseados em conceitos. Ele demonstrou tal afirmação ao apresentar palavras em um contexto neutro ou previsível (ex: "o aluno entediado abriu sua boca para... bocejar"). Palavras que apareciam junto a contextos previsíveis eram externalizadas mais rapidamente, indicando maior fluência de processamento.

Posteriormente, os participantes avaliaram contextos previsíveis como mais agradáveis. O autor então concluiu afirmando que a fluência conceitual é definida pelo contexto da situação e pela previsibilidade semântica do estímulo. Para Whittlesea (1993), apenas esse tipo de fluência pode influenciar julgamentos sobre significados, a menos que algo leve o indivíduo a interpretar erroneamente a fluência perceptual como sendo fluência conceitual. Embora ambos tipos sejam consequências naturais da exposição, a fluência conceitual é mais afetada pela quantidade de exposições anteriores (Janiszewski \& Meyvis, 2001). Lee e Labroo (2004) ampliaram os resultados de Reber et al. (1998) da fluência perceptiva para a fluência conceitual sobre a valência positiva de um estímulo neutro. No entanto, para a fluência conceitual, se a marca é apresentada em um contexto negativo (ex: um shampoo que diz "não ser bonzinho com piolhos"), ou seja, um processamento conceitualmente fluente está associado a construtos de valência negativa, um efeito negativo pode ocorrer, como diminuir a atitude do consumidor em relação à marca.

Apesar de ambas serem ativadas através de exposições prévias, a fluência perceptual e a conceitual são processos independentes, com diferentes antecedentes e consequências (Cabeza \& Ohta, 1993; Lee, 2002). Por exemplo, a fluência perceptual é mais sensível a mudanças em características superficiais como trocas de modos (auditiva $\mathrm{x}$ visual) e contextos de apresentação (tarefa de reconhecimento x tarefa de memória) (Jacoby \& Dallas, 1981), além de não ser afetada pela elaboração, isto é, a quantidade de pensamento (Bohner \& Dickel, 2011), um fator importante somente para a fluência conceitual na mudança de atitude do consumidor (Eich, 1984; Hamann, 1990). A principal diferença da fluência conceitual para a perceptual é sua capacidade de resgatar estímulos em contextos relacionados a eles sem a necessidade de ser exposto novamente ao estímulo (Nedungadi, 1990). Um exemplo disso é o impacto positivo que um priming indireto (ex: marca concorrente) pode exercer sobre o indivíduo, aumentando a fluência do estímulo-alvo (Lee \& Labroo, 2004).

Dessa forma, pode-se dizer que a fluência de processamento, perceptual e conceitual, caminha junto com a familiaridade. Estímulos familiares não são apenas processados mais rapidamente (Jacoby \&
Dallas, 1981), como possuem uma dinâmica de processamento mais organizada do que estímulos inéditos (Smith, 2000). Estímulos familiares transmitem segurança (Zajonc, 1968), uma necessidade básica do ser humano que pode ser obtida em reencontros entre o indivíduo e um estímulo conhecido no passado. Contudo, a sensação familiar também pode surgir sem que haja uma experiência prévia com o estímulo e, desde que o processamento desse estímulo seja fluente (Whittlesea, 1993), isto ocorre porque segundo Jacoby, Kelley e Dywan (1989), a fluência de processamento atribui sensações de familiaridade à fonte mais provável de ser a causa da fluência. Essa sensação de familiaridade só emergirá se a fonte mais provável estiver no passado, caso ela seja atribuída a algo no presente, a sensação gerada é a de qualidade (Whittlesea, 1993).

Por fim e para uma melhor compreensão do tema, nas seções seguintes do artigo, será usado fluência como sinônimo para fluência de processamento que, por sua vez, abrange a fluência perceptual e fluência conceitual. Uma vez que a distinção entre os dois tipos não é relevante para muitos dos estudos no contexto do consumidor devido à similaridade dos efeitos. Seguindo Tsai e McGill (2011), será utilizado o tipo específico apenas quando os efeitos divergirem ou a distinção for recomendável para melhor compreensão.

\subsection{Teorias que Explicam a Fluência}

Há três principais teorias para explicar a fluência: atribuição errônea (misattribution model ${ }^{v}$ ), redução da incerteza (uncertainty reduction ${ }^{v i}$ ) ou modelo de dois fatores modificado (modified twofactor model ${ }^{v i i}$ ) e teoria do processo duplo (dualprocess theory ${ }^{\text {viii }}$ ).

Segundo Schwarz (2004), as pessoas desenvolvem teorias ingênuas sobre as causas das experiências fluentes, de modo que nenhuma das justificativas fornecidas por elas para justificar seu julgamento será a verdadeira, isto é, o fato de já terem experienciado o estímulo anteriormente. Esta é a premissa por trás do modelo de atribuição errônea, a teoria mais utilizada para explicar os efeitos da fluência (Bornstein \& D'Agostino, 1994; Dechene, Stahl, Hansen, \& Wanke, 2010; McGlone \& Tofighbakhsh, 2000; Reber et al., 1998; Shapiro, 1999; Tanner \& Maeng, 2012). A fluência de processamento considera que um estímulo visto anteriormente será mais facilmente percebido, codificado e processado em encontros futuros (Bornstein \& D’Agostino, 1992, 1994), entretanto, os indivíduos falham em reconhecer que a sensação de facilidade é resultado da fluência gerada por exposições anteriores; assim, desde que o contexto contribua para a plausibilidade do julgamento, a 
sensação agradável de facilidade é erroneamente atribuída a outros aspectos como maior preferência, familiaridade, beleza ou aceitabilidade da fonte (Janiszewski \& Meyvis, 2001; Klinger \& Greenwald, 1994).

Como a fluência de processamento é um processo da memória implícita, seus efeitos ocorrerão apenas quando os indivíduos não conseguirem lembrar conscientemente a real causa da fluência, ou seja, de já terem visto o estímulo anteriormente. Ao se lembrar de forma consciente (um processo da memória explícita) de experiências anteriores com o estímulo, as justificativas entram em conflito. Em outras palavras, se o indivíduo tem consciência que está sendo manipulado pela fluência, ele automaticamente corrige o viés da fluência e passa a atribui-la não às suas consequências (preferência, familiaridade, beleza, confiabilidade, etc.), mas ao fato de já terem sido expostos ao estímulo. Dessa forma, a fluência perde seus efeitos (Bornstein \& D'Agostino, 1992; Janiszewski \& Meyvis, 2001). Um exemplo de mal atribuição é a música de fundo (Mishra, Mishra, \& Nayakankuppam, 2006); em um estudo sobre a tendência das pessoas em poupar notas maiores e gastar mais notas menores ( $\$ 100$ vs $\$ 50, \$ 20, \$ 20$, $\$ 10)$, indivíduos informados de que a música ambiente poderia influenciar seus sentimentos sobre o dinheiro, de fato, tiveram sua percepção de valor de uma nota de $\$ 50$ reduzida, enquanto os que não receberam essa informação, valorizaram mais a nota de $\$ 50$. Isso acontece porque a reação afetiva passa a ser atribuída erroneamente à música e não ao dinheiro inteiro ( $\$ 50 \mathrm{vs} \mathrm{dez} \mathrm{notas} \mathrm{de} \$ 5$ ) que é um objeto fluente. Por fim, é importante destacar que tanto as atribuições errôneas quanto a correção do viés são inconscientes e automáticos (Bornstein \& D’Agostino, 1992).

Uma segunda tentativa de explicar os efeitos da fluência é através do modelo de doisfatores modificado, também chamado de redução da incerteza. Ele defende que as respostas afetivas são funções do aprendizado e da saciedade (Berlyne, 1970); estes dois, por sua vez, são a fonte da fluência (Janiszewski \& Meyvis, 2001). O aprendizado reduz a incerteza que leva a efeitos positivos sobre um estímulo, enquanto a saciedade gera tédio e, consequentemente, efeitos negativos. Dessa forma, repetições levam a uma redução da incerteza e do conflito, melhorando a apreciação do estímulo. Por outro lado, repetições também aumentam o tédio, o que reduz a apreciação. Diferente da atribuição errônea que diz ser a má atribuição a causa do efeito, a modelo de dois fatores modificado defende que é a familiaridade subjetiva gerada pela exposição prévia a causa do efeito da fluência (Lee, 2001).

Portanto, a familiaridade possui um papel fundamental para a redução da incerteza / modelo de dois fatores modificado. Enquanto na atribuição errônea, se a experiência anterior for lembrada, há uma correção pra excluí-la da avaliação, na redução da incerteza essa familiaridade subjetiva gera efeitos positivos. Nesse caso, achar que o objeto já foi apresentado ao indivíduo tem vantagem sobre o não apresentado. Sendo assim, como demonstrou Lee (2001), o estímulo novo cujo participante não se lembrava de já tê-lo visto foi o pior avaliado segundo a redução da incerteza, enquanto na atribuição errônea o pior avaliado foi o estímulo novo cujo participante se lembra de já o ter visto. Todavia, a semelhança entre as duas teorias é que, em ambas, o antigo foi preferido sobre o novo, o que demonstra a força do efeito da fluência (Lee, 2001) apesar do confito de explicações.

Por fim, a teoria do processo duplo pode ser considerada uma terceira explicação para os efeitos positivos gerados da fluência. Ela considara que as respostas afetivas são funções de sensibilização e habituação. A sensibilização é uma resposta não específica e sensível à significância e à intensidade do estímulo (estímulos complexos como novidades e imagens com alto-contraste são mais estimulantes). Já a habituação é inibitória e específica do sistema nervoso e aumenta a cada exposição, porém diminui com o tempo. A habituação também é afetada pela intensidade, porém, quanto menos intenso for, mais rápido será a habituação, e também pelo tempo de intervalo das exposições. Exposições repetidas aceleram a habituação, enquanto diminuem a sensibilização (Groves \& Thompson, 1970). O processo duplo difere da redução da incerteza ao considerar um processamento passivo das informações, onde cada estímulo tem seu nível de significância que não é alterado pela experiência. Para a redução da incerteza, indivíduos investigam ativamente um estímulo novo na busca por diminuir sua incerteza e aumentar o seu conforto em relação ao estímulo (Janiszewski \& Meyvis, 2001).

Para exposições de marketing, Janiszewski e Meyvis (2001) afirmam que a teoria do processo duplo é mais adequada para explicar exposições repetidas a estímulos estáticos (marcas, logos, embalagens), enquanto o modelo de dois fatores modificado é mais indicado para explicar exposições repetidas a comerciais de TV. Mais importante, os autores consideraram a teoria do processo duplo mais compatível com o modelo de atribuição errônea, que é a explicação mais popular entre os pesquisadores para explicar os efeitos da fluência. Sendo assim, o modelo de dois fatores modificado/redução da incerteza é o mais distinto dentre os três, e há indícios de que o fenômeno da fluência é melhor explicado por este modelo do que pela atribuição errônea (Lee, 2001), ainda assim este último permanece como a explicação mais utilizada pelos pesquisadores. Mais estudos parecem ser 
necessários para se ter certeza qual a melhor explicação para a fluência.

Outra crítica ao modelo de atribuição errônea éa sua valência neutra. Segundo este modelo, é a atribuição errada (a incapacidade do indivíduo de perceber que já fora exposto ao estímulo o leva a atribuir seus julgamentos a outros fatores e não às exposições prévias com o estímulo) a causa da sensação agradável que a fluência proporciona. Se não fosse pela má atribuição, o impacto de um estímulo fluente seria neutro. Ou seja, para o modelo de atribuição errônea a fluência é naturalmente neutra (é a atribuição da fluência que gera efeitos positivos). Todavia, há evidências robustas para acreditar na natureza positiva da fluência (Lee \& Labroo, 2004; Reber et al., 1998; Stang, 1974), o que a torna mais compatível com o modelo de dois fatores e redução da incerteza que considera que a fluência é naturalmente positiva (Lee, 2001).

\subsection{Multitarefa de mídia}

A tecnologia sempre demonstrou ter a capacidade de moldar a sociedade e o comportamento das pessoas ao longo do tempo. A chegada dos computadores, considerada a quinta grande onda tecnológica (Mackey, Freeman, \& Oil, 1998), gerou a revolução da informação e provocou mudanças profundas na dinâmica da sociedade, facilitando não apenas $\mathrm{o}$ processamento $\mathrm{e}$ armazenamento de informações, mas também aumentando drasticamente a eficácia da comunicação. Com o tempo, a popularização e a sofisticação dos computadores parece ter gerado uma "sociedade acelerada", dotada de uma nova percepção de tempo e espaço (Wajcman, 2008). Assim, indivíduos buscam extrair a máxima produtividade de um fragmento de tempo, o que aumenta as chances de se engajarem em comportamentos multitarefa (multitasking), que podem ser definidos como a realização simultânea de duas ou mais atividades com finalidades distintas (Sanbonmatsu, Strayer, Medeiros-Ward, \& Watson, 2013).

A pervasividade da tecnologia na forma de diferentes dispositivos e mídias deu origem, sobretudo na última década, a um novo tipo de multitarefa, a multitarefa de mídia (media multitasking $\left.{ }^{i x}\right)$. Multitarefa de mídia é o engajamento ou consumo simultâneo de duas ou mais mídias, sejam estas online ou offline (Bardhi, Rohm, \& Sultan, 2010; Foehr, 2006; Pilotta, Schultz, Drenik, \& Rist, 2004; Voorveld, 2011).

A multitarefa de mídia difere da multitarefa tradicional em dois aspectos: 1) a natureza interativa do conteúdo frequentemente demanda respostas imediatas por parte do indivíduo em multitarefa; 2) a estrutura do fluxo de informações de algumas mídias requer atenção constante para ser assimilada (Kazakova, Pandelaere, \& De Pelsmacker, 2015). Este comportamento está se tornando o padrão em pessoas de todas as idades (Voorveld \& Goot, 2013) e parece ter o poder de afetar a efetividade de mensagens publicitárias. Estudos sugerem que realizar mais de uma atividade ao mesmo tempo diminui a lembrança e reconhecimento do conteúdo exibido (Angell, Gorton, Sauer, Bottomley, \& White, 2016; Bellman, Robinson, Wooley, \& Varan, 2014; Bellman, Rossiter, Schweda, \& Varan, 2012; Brasel \& Gips, 2011; Voorveld, 2011). Por exemplo, ler jornal enquanto o televisor está ligado diminui a lembrança e compreensão das informações (Armstrong \& Chung, 2000) e o mero ato de analisar fotografias com o rádio ligado reduz as respostas favoráveis dos indivíduos em relação a um comercial veiculado durante a tarefa (Bolls \& Muehling, 2007).

Kazakova, Cauberghe, Hudders e Labyt (2016) demonstraram que participantes que assistiram um comercial enquanto navegam em um website relataram menor lembrança espontânea e reconhecimento da marca exposta, em comparação aos participantes que somente assistiram ao comercial. O estudo também demonstrou que produtos que apelavam ao desejo (contra utilidade) geraram maior atitude dos participantes em multitarefa, enquanto não houve diferença entre os participantes expostos a uma única mídia. Os autores explicam que o apelo a sensações positivas de posse (desejo) requer menos esforço cognitivo do que o apelo aos atributos do produto (utilidade), facilitando o processamento e, como consequência, levando a uma atitude mais positiva.

Três modelos ajudam a entender os efeitos potenciais da multitarefa de mídia nas mensagens publicitárias. O primeiro é o modelo de persuasão (McGuire, 1985), que diz que para a persuasão de uma mensagem ocorrer, a exposição não é suficiente, sendo necessário atenção, compreensão e aceitação. A multitarefa, contudo, pode restringir a atenção ou a compreensão, devido à limitada capacidade de processamento do ser humano, conforme sugere o segundo modelo, o de processamento de informação (Best, 1986; Lang, 2000), que defende que tarefas mais complexas demandam mais recursos, reduzindo a atenção e compreensão do conteúdo das mensagens. O terceiro modelo é o Elaboration Likelihood Model (Petty; Cacioppo; Schumann, 1983), que afirma que algumas tarefas geram distração que podem reduzir a habilidade do consumidor de processar o conteúdo da mensagem. Como consequência, indivíduos não processarão as mensagens pela rota central, e sim pela rota periférica, o que pode reduzir os efeitos da mensagem nas atitudes e intenções a longo prazo. Dessa forma, pode-se inferir que a multitarefa de 
mídia pode reduzir a efetividade de mensagens de marketing, diminuindo a atenção do consumidor e o fazendo processar tais mensagens mais superficialmente (Voorveld, 2011).

\section{PROPOSIÇÕES TEÓRICAS}

Para auxiliar a condução de estudos futuros sobre fluência de processamento e ampliar a literatura sobre os efeitos do comportamento multitarefa de mídia na efetividade de estímulos de marketing,este artigo propõe um modelo teórico- empírico de estudo (Figura 1) para analisar os dois tipos de fluência de processamento,fluência perceptual e fluência conceitual, no contexto multitarefa Vale ressaltar que a fluência perceptual e conceitual embora sejam geradas pela exposição prévia com o estímulo são construtos independentes (Cabeza \& Ohta, 1993) e possuem diferentes antecedentes e consequentes (Lee, 2002). Há razões para acreditar que mensagens de marketing podem aumentar sua eficácia combinando os dois tipos de fluência (Hang \& Auty, 2011), sobretudo, em contextos de pouca atenção ou alto esforço cognitivo como multitarefa de mídia.

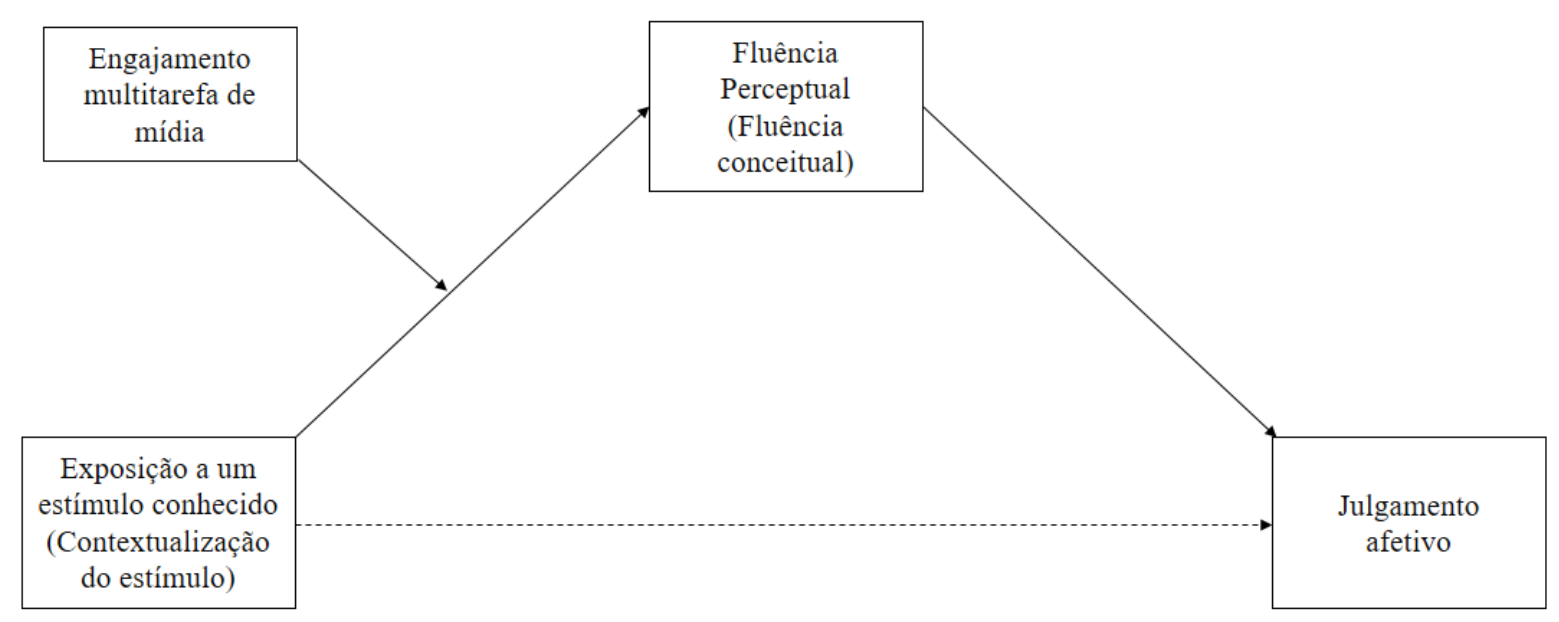

Figura 1 - Modelo teórico

Fonte: Elaborado pelos autores

A multitarefa de mídia é especialmente importante para a fluência, pois pode alterar a forma como espectadores processam conteúdo midiático (Kazakova et al., 2015), pois ambasenvolvem processos cognitivos relacionados ao processamento de informação. Considerando que o excesso de ruído sempre foi um entrave para a eficiência das mensagens publicitárias (Kent, 1995; Riebe \& Dawes, 2006) e a capacidade limitada de processamento do ser humano (Best, 1986), entender como a fluência funciona em um contexto de multitarefa poderá ajudar as empresas a eliminar ruídos da comunicação e fazer com que a mensagem não apenas chegue até o seu destino, mas seja processada de forma a gerar reações positivas.

Segundo a fluência de processamento, a mente humana armazena uma grande coleção de exposições a estímulos, de forma a facilitar o seu processamento no próximo encontro (Bornstein \& D’Agostino, 1994; Janiszewski, 1993). Dependendo do tipo de estímulo, o processamento facilitado (fluência) futuro pode ser de dois tipos: perceptual ou conceitual. Fluência perceptual é a facilidade, gerada por uma exposição prévia, com que o indivíduo processa características físicas de um estímulo (Jacoby \& Dallas, 1981). Enquanto a fluência perceptual precisa de um novo encontro para ser ativado, a conceitual não. Nesse caso, a exposição prévia a um contexto cria uma representação mental de significado que facilitará a codificação e o processamento do estímulo alvo (Shapiro, 1999; Whittlesea, 1993). Por exemplo, ao ver uma lanchonete lotada e um hambúrguer sendo preparado, aumenta a fluência conceitual para uma marca de ketchup ou maionese, uma vez que esses produtos são mais fáceis de prever, pois possuem maiores associações entre si. O mesmo não acontece ao ver uma mulher em uma gôndola de supermercado (Lee \& Aaker, 2004). Se o indivíduo possuir uma marca de ketchup ou maionese perceptualmente fluente, há chances dele pensar 
nessa marca específica ao ser exposto à cena da lanchonete.

Os dois tipos de fluências são estimulados pela repetição (Whittlesea, 1993), mas apenas a fluência conceitual envolve significados (Whittlesea \& Williams, 2000), o que a torna menos sensível a mudanças de características do estímulo, por exemplo o modo de apresentação (sonoro vs visual) e mais sensível à natureza preditiva do contexto em que o estímulo é apresentado. Além disso, para a fluência perceptual $o$ estímulo precisa ser apresentado o mais parecido possível com o que foi visto anteriormente (Shapiro, 1999), por exemplo, se foi o anúncio mostra o produto fora da caixa, a fluência será maior se no ponto de venda o consumidor vê-lo na caixa ou a embalagem exibir o produto de forma clara e direta. Embora seja a fluência como um todo (fluência de processamento) que importa para o marketing, isto é significados e atributos visuais juntos, e seus efeitos combinados sejam mais fortes (Lee \& Labroo, 2004), a fluência perceptual e conceitual são independentes (Cabeza \& Ohta, 1993). Diante disso, este artigo propõe que encontros com estímulos de marketing variados (marcas, produtos, anúncios) aumentarão a fluência desses estímulos e, consequentemente, o julgamento afetivo do consumidor por eles em um novo encontro. O tipo de fluência gerada irá depender da existência de um contexto em torno do estímulo e se este contexto está de acordo com a estrutura de conhecimento semântico do consumidor.

$\mathrm{P}_{1 \mathrm{a}}$ : Exposição anterior a um estímulo de marketing irá aumentar o julgamento afetivo deste estímulo.

$\mathrm{P}_{1 \mathrm{~b}}$ : Exposição a um estímulo de marketing contextualizado irá aumentar o julgamento afetivo deste estímulo.

Um dos principais objetivos de marketing é o aumento da conscientização da marca, ela é importante para que o consumidor possa incluí-la entre as opções de compra e seja levado a adquirir o produto ou serviço daquela marca (Macdonald \& Sharp, 1996, 2000). Nedungadi (1990) mostrou que a alta acessibilidade da marca na mente leva a maior inclusão dela entre as opções de compra, no entanto sem aumentar a avaliação da marca, mostrando que um julgamento afetivo positivo não é o único viés utilizado na decisão de compra. Shapiro (1999) expandiu o achado creditando tal efeito à fluência. A natureza afetiva positiva da fluência (Lee \& Labroo, 2004; Reber et al., 1998) faz com que qualquer estímulo neutro fluente receba avaliações mais positivas do que um estímulo não fluente, além de ser considerado como mais verdadeiro, aceitável, bonito, famoso e familiar (Begg et al., 1992; Reber, Schwarz, \& Winkielman, 2004; Tanner \& Maeng,
2012; Whittlesea \& Williams, 2000). Apesar disso, julgamentos cognitivos (beleza, fama, risco, etc.) não fazem parte do modelo teórico proposto que limita-se a julgamentos afetivos (preferência, apreciação, atratividade, agradabilidade, etc.), uma vez que indivíduos tendem a não corrigir respostas afetivas ao contrário das cognitivas (Lee, 2001); forte relação do julgamento afetivo com o consumo (Brown \& Stayman, 1992; Westbrook, 1980), e amplo uso nos estudos de fluência (Oppenheimer, 2008; Reber et al., 2004, 1998; Winkielman, Schwarz, Fazendeiro, \& Reber, 2003). Vários estudos ligam a fluência a melhor avaliação do estímulo (Janiszewski, 1990; McCullough \& Ostrom, 1974; Mishra et al., 2006; Reber et al., 1998; Schwarz, 2004; Winkielman, Berridge, \& Wilbarger, 2005), tornando-a um importante aspecto no comportamento do consumidor (Chang, 2013). Dessa forma, o modelo teórico proposto abrange dois fortes fatores que influenciam a escolha do consumidor por uma marca, seja através de uma relação direta entre o estímulo e o julgamento afetivo ou indireta mediada pela fluência.

$\mathrm{P}_{2 \mathrm{a}}$ : A fluência perceptual irá mediar os efeitos das exposições anteriores no aumento do julgamento afetivo.

$\mathrm{P}_{2 \mathrm{~b}}$ : A fluência conceitual irá mediar os efeitos da contextualização no aumento do julgamento afetivo.

Contudo, a fluência pode ter seus efeitos reduzidos pelo recente padrão de comportamento multitarefa que vem sendo estimulado, em grande parte, pela tecnologia. Ainda que um milissegundo seja suficiente para um estímulo previamente exposto aumentar a preferência do indivíduo (KunstWilson \& Zajonc, 1980) e um anúncio na visão periférica de um leitor aumentar a atitude dele em relação ao anúncio (Janiszewski, 1990, 1993), e que o efeito de mera exposição é maior em estímulos inconscientes do que conscientes (Bornstein, 1989; Bornstein \& D’Agostino, 1992), a relação entre engajamento multitarefa e fluência de processamento demanda estudos. Realizar mais de uma tarefa ao mesmo tempo diminui a retenção de informações (Lee, Lin, \& Robertson, 2012), o estudo de Kazakova et al. (2015) mostra que o engajamento de mídia modifica a forma como os indivíduos processam as informações dessas mídias e chama atenção para o processamento cognitivo e afetivo do conteúdo midiático e persuasivo típico da propaganda. Diante disso e do fato do consumo de mídia ser cognitivamente exigente (Bardhi, Rohm, \& Sultan, 2010), é possível que o engajamento multitarefa dificulte $\mathrm{o}$ processamento de informações visual, especialmente no público jovem que é o maior adepto da prática. A multitarefa de 
mídia, portanto, deve exercer um papel moderador na exposição e diminuindo a fluência gerada pelo estímulo.

A escolha por um produto ou marca não é baseada exclusivamente em informações, mas também na facilidade e quantidade de informação que os indivíduos precisam processar para decidir ( Lee \& Labroo, 2004). Muita informação tende a desencorajar a decisão (Iyengar \& Lepper, 2000), o que pode ajudar a explicar o fato de pessoas tomarem decisões com base na facilidade com que conseguem processar (Tversky \& Kahneman, 1973). Desse modo, em uma época onde as pessoas prestam cada vez menos atenção e realizam várias atividades simultâneas, especialmente enquanto consomem mídia (Rideout, Foehr, \& Roberts, 2010), se os efeitos da fluência ainda ocorrerem durante $\mathrm{o}$ engajamento multitarefa de mídia, empresas terão um artifício a seu favor. Com base na literatura (Lee \& Labroo, 2004; Novemsky et al., 2007; Seamon et al., 1995), é possível inferir que estímulos de marketing fluentes serão mais facilmente processados e obterão avaliações mais positivas do que estímulos de marketing não fluentes mesmo enquanto exercem multitarefa.

Buscando expandir os efeitos da fluência para o contexto digital, utilizando o modo de comportamento típico do meio tecnológico (Voorveld \& Goot, 2013), propõe-se:

$\mathrm{P}_{3 \mathrm{a}}$ : O engajamento multitarefa irá moderar os efeitos das exposições anteriores na fluência perceptual.

$\mathrm{P}_{3 \mathrm{~b}}$ : O engajamento multitarefa de mídia irá moderar os efeitos da contextualização na fluência conceitual.

A partir do efeito de mera exposição que diz que a simples exposição a um estímulo aumenta a avaliação positiva do indivíduo em relação ao estímulo (Zajonc, 1968), Janiszewski et al. (2013) argumentam que apenas olhar para o estímulo não é o suficiente para levar o consumidor à escolha, é preciso que haja atenção para aumentar as chances de um produto ser escolhido no futuro. Produtos que não receberam atenção (foco), mas estavam presentes tiveram menos chances de serem escolhidos no futuro. Segundo o estudo, a atenção seletiva ajuda a prever escolha, sobretudo em um ambiente com múltiplas alternativas e em situações em que há frequentes trocas de modos atenção/desatenção. Isso torna o engajamento multitarefa de mídia mais prejudicial à mensagem publicitária, uma vez que reduz o foco em um único objeto através de constantes trocas de mídias.

Por fim, vale alertar para a consciência do efeito do estímulo, um grande perigo à fluência que pode reduzir ou eliminar totalmente seus (Bornstein
\& D’Agostino, 1994). De acordo com o modelo de atribuição errônea, explicação mais popular para a fluência, a apresentação repetida de um estímulo gera uma sensação de facilidade (fluência) quando o objeto é encontrado novamente (Bornstein \& D’Agostino, 1994; Jacoby et al., 1989). Esta sensação então é mal atribuída a algum fator como o fato de gostar, ser verdadeiro ou confiável (Whittlesea, 1993). No entanto, quando o indivíduo se dá conta do estímulo e que esse pode ter influenciado o seu julgamento, o indivíduo automaticamente corrige o viés, atribuindo seu julgamento às exposições anteriores. Em ambos os casos, isto é percebendo ou não exposição prévia, o processo de atribuição ocorre automaticamente e sem esforço (Bornstein \& D’Agostino, 1994; Klinger \& Greenwald, 1994). A fluência parece funcionar melhor em situações low level processing (Dechene et al., 2010), ou seja, se algo chamar a atenção do consumidor, levando-o a refletir sobre a mensagem, o efeito da fluência diminuirá ou desaparecerá. Nesse caso, reações afetivas declarativas podem sobrepor reações afetivas experienciais (Winkielman et al., 2003), em um clássico duelo entre razão e emoção.

\section{CONSIDERAÇÕES FINAIS}

A influência da exposição prévia a um estímulo e o aumento de atitudes favoráveis em relação a ele em um encontro posterior tem sido documentada há bastante tempo (Bornstein, 1989; Maslow, 1937; Zajonc, 1968). Seus efeitos robustos já foram demonstrados com pinturas, palavras, ideogramas chineses, sílabas desconexas, rostos, roupas, cheiros, comidas e sons (Bornstein, 1989; Capaldi, 1992; Peretz, Gaudreau, \& Bonnel, 1998; Porter \& Winberg, 1999; Tanner \& Maeng, 2012). Encontros prévios aumentam a facilidade com que um estímulo é processado, isto é, a facilidade em perceber, codificar, compreender e recuperar informações, que podem gerar dois tipos de fluência: perceptual (representação visual do estímulo) e conceitual (representação do estímulo baseada em significados). Essa fluidez de processamento é capaz de influenciar a preferência por uma opção (Schwarz, 2004), e como tem mostrado os estudos das últimas duas décadas, também são capazes de influenciar consumidores em um processo que é tanto consciente como inconsciente (Jacoby, 1991; Janiszewski \& Meyvis, 2001).

Estudos de fluência de processamento na propaganda já foram realizados em variados ambientes, com diferentes estímulos e em diversas situações, mas nenhum investigou seus efeitos em um diferente tipo de comportamento que está se tornando o padrão da sociedade cada vez mais 
dinâmico e tecnológico (Voorveld \& Goot, 2013), o comportamento multitarefa. A partir de uma breve revisão da literatura da fluência, este artigo propôs um modelo teórico de pesquisa com o objetivo de orientar futuros estudos de fluência de processamento no comportamento do consumidor considerando uma variável moderadora inédita, o engajamento multitarefa de mídia. Para isso, sugerese a realização de pesquisas experimentais em laboratório.

Espera-se que este artigo possa contribuir para o avanço dos estudos da fluência e que possa atrair interesse dos pesquisadores brasileiros para um tema tão relevante.

\section{REFERÊNCIAS}

Angell, R., Gorton, M., Sauer, J., Bottomley, P., \& White, J. (2016). Don't Distract Me When I'm Media Multitasking: Toward a Theory for Raising Advertising Recall and Recognition. Journal of Advertising, 45(2), 1-13. http://doi.org/10.1080/00913367.2015.1130665

Armstrong, G. B., \& Chung, L. (2000). Background Television and Reading Memory in Context: Assessing TV Interference and Facilitative Context Effects on Encoding Versus Retrieval Processes. Communication Research, 27, 327-352. http://doi.org/10.1177/009365000027003003

Bardhi, F., Rohm, A. J., \& Sultan, F. (2010). Tuning in and tuning out: Media multitasking among young consumers. Journal of Consumer Behaviour, 9(4), 316-332.

http://doi.org/10.1002/cb.320

Begg, I. M., Anas, A., \& Farinacci, S. (1992). Dissociation of processes in belief: Source recollection, statement familiarity, and the illusion of truth. Journal of Experimental Psychology: General, 121(4), 446-458.

http://doi.org/10.1037/0096-3445.121.4.446

Bellman, S., Robinson, J. a., Wooley, B., \& Varan, D. (2014). The effects of social TV on television advertising effectiveness. Journal of Marketing Communications, (July 2015), 1-19. http://doi.org/10.1080/13527266.2014.921637

Bellman, S., Rossiter, J. R., Schweda, A., \& Varan, D. (2012). How coviewing reduces the effectiveness of TV advertising. Journal of Marketing Communications, 18(5), 363-378. http://doi.org/10.1080/13527266.2010.531750

Berlyne, D. E. (1970). Novelty, complexity, and hedonic value. Perception \& Psychophysics, 8(5), 279-286. http://doi.org/10.3758/BF03212593

Best, J. B. (1986). Cognitive Psychology (5th ed.). Wiley.

Bohner, G., \& Dickel, N. (2011). Attitudes and Attitude Change. Annual Review of Psychology, $62,391-417$.

http://doi.org/10.1146/annurev.psych.121208.13160 9

Bolls, P. D., \& Muehling, D. D. (2007). The Effects of Dual-Task Processing on Consumers' Responses to High- and Low-Imagery Radio Advertisements. Journal of Advertising, 36(4), 35-47.

Bornstein, R. F. (1989). Exposure and affect: Overview and meta-analysis of research 1968-1987. Psychological Bulletin, 106(2), 265-289.

Bornstein, R. F., \& D’Agostino, P. R. (1992). Stimulus recognition and the mere exposure effect. Journal of Personality and Social Psychology, 63(4), 545-552. http://doi.org/10.1037/00223514.63.4.545

Bornstein, R. F., \& D’Agostino, P. R. (1994). The Attribution and Discounting of Perceptual Fluency: Preliminary Tests of a Perceptual Fluency/Attributional Model of the Mere Exposure Effect. Social Cognition, 12(2), 103-128. http://doi.org/10.1521/soco.1994.12.2.103

Brasel, S. A., \& Gips, J. (2011). Media Multitasking Behavior: Concurrent Television and Computer Usage. Cyberpsychology, Behavior, and Social Networking, 14(9), 527-534. http://doi.org/10.1089/cyber.2010.0350

Brown, S. P., \& Stayman, D. M. (1992). ANTECEDENTS AND CONSEQUENCES OF ATTITUDE TOWARD THE AD - A METAANALYSIS. Journal of Consumer Research, 19(1), 34-51. http://doi.org/10.1086/209284

Cabeza, R., \& Ohta, N. (1993). Dissociating conceptual priming, perceptual priming and explicit memory. European Journal of Cognitive Psychology, 5(1), 35-53. http://doi.org/10.1080/09541449308406513

Capaldi, E. D. (1992). Conditioned Food Preferences. Psychology of Learning and Motivation - Advances in Research and Theory, 28(C), 1-33. http://doi.org/10.1016/S00797421(08)60486-7 
Chae, B. (Grace), \& Hoegg, J. (2013). The Future Looks "Right": Effects of the Horizontal Location of Advertising Images on Product Attitude. Journal of Consumer Research, 40(August), 223-238. http://doi.org/10.1086/669476

Chang, C. J. (2013). Price or quality? The influence of fluency on the dual role of price. Marketing Letters, 24(4), 369-380. http://doi.org/10.1007/s11002-013-9223-8

Dechene, A., Stahl, C., Hansen, J., \& Wanke, M. (2010). The Truth About the Truth: A MetaAnalytic Review of the Truth Effect. Personality and Social Psychology Review, 14(2), 238-257. http://doi.org/10.1177/1088868309352251

Eich, E. (1984). Memory for unattended events: Remembering with and without awareness. Memory \& Cognition, 12(2), 105-111. http://doi.org/10.3758/BF03198423

Foehr, U. G. (2006). Media multitasking among American youth: Prevalence, predictors, and pairings. The Henry J. Kaiser Family Foundation, (December), 1-39. http://doi.org/10.1111/j.14602466.2012.01641.x

Groves, P. M., \& Thompson, R. F. (1970). Habituation: A dual-process theory. Psychological Review, 77(5), 419-450. http://doi.org/10.1037/h0029810

Hamann, S. B. (1990). Level-of-Processing Effects in Conceptually Driven Implicit Tasks. Journal of Experimental Psychology. Learning, Memory \& Cognition, 16(6).

Hang, H., \& Auty, S. (2011). Children playing branded video games: The impact of interactivity on product placement effectiveness. Journal of Consumer Psychology, 21(1), 65-72. http://doi.org/10.1016/j.jcps.2010.09.004

Iyengar, S. S., \& Lepper, M. R. (2000). When choice is demotivating: Can one desire too much of a good thing? Journal of Personality and Social Psychology, 79(6), 995-1006. http://doi.org/10.1037/0022-3514.79.6.995

Jacoby, L. L. (1991). A process dissociation framework: Separating automatic from intentional uses of memory. Journal of Memory and Language, 30(5), 513-541. http://doi.org/10.1016/0749596X(91)90025-F

Jacoby, L. L., \& Dallas, M. (1981). On the relationship between autobiographical memory and perceptual learning. Journal of Experimental Psychology, 110(3), 306-340.

http://doi.org/10.1037/0096-3445.110.3.306

Jacoby, L. L., Kelley, C. M., \& Dywan, J. (1989). Memory attributions. In Henry L. Roediger \& F. I. M. Craik (Eds.), Varieties of memory and consciousness Essays in honour of Endel Tulving (Vol. 57, pp. 391-422). Psychology Press. http://doi.org/10.1016/j.jml.2006.08.012

Jacoby, L. L., Woloshyn, V., \& Kelley, C. (1989). Becoming famous without being recognized: Unconscious influences of memory produced by dividing attention. Journal of Experimental Psychology: General, 118(2), 115-125. http://doi.org/10.1037/0096-3445.118.2.115

Janiszewski, C. (1990). The Influence of Print Advertisement Organization on Affect toward a Brand Name. Journal of Consumer Research, 17(1), 53. http://doi.org/10.1086/208536

Janiszewski, C. (1993). Preattentive mere exposure effects. Journal of Consumer Research,

20(December), 376-392. http://doi.org/10.1086/209356

Janiszewski, C., Kuo, A., \& Tavassoli, N. T. (2013). The influence of selective attention and inattention to products on subsequent choice. Journal of Consumer Research, 39(6), 1258-1274. http://doi.org/10.1086/668234

Janiszewski, C., \& Meyvis, T. (2001). Effects of Brand Logo Complexity, Repetition, and Spacing on Processing Fluency and Judgment. Journal of Consumer Research, 28(1), 18-32. http://doi.org/10.1086/321945

Kazakova, S., Cauberghe, V., Hudders, L., \& Labyt, C. (2016). The Impact of Media Multitasking on the Cognitive and Attitudinal Responses to Television Commercials : The Moderating Role of Type of Advertising Appeal, $0(0), 1-14$.

http://doi.org/10.1080/00913367.2016.1183244

Kazakova, S., Pandelaere, M., \& De Pelsmacker, P. (2015). Can't See the Forest for the Trees? The Effect of Media Multitasking on Cognitive Processing Style. Media Psychology, 18, 425-450. http://doi.org/10.1080/15213269.2015.1006789

Kent, R. J. (1995). Competitive Clutter In Network Television Advertising: Current Levels And Advertiser Responses. Journal of Advertising Research, 35(1), 49-57. 
Kim, H., Rao, A. R., \& Lee, A. Y. (2009). It's Time to Vote: The Effect of Matching Message Orientation and Temporal Frame on Political Persuasion. Journal of Consumer Research, 35(6), 877-889. http://doi.org/10.1086/593700

Klinger, M. R., \& Greenwald, A. G. (1994). Preferences need no inferences?: The cognitive basis of unconscious mere exposure effects. In The heart's eye: Emotional influences in perception and attention (pp. 67-85).

Kramer, T., \& Kim, H. M. (2007). Processing fluency versus novelty effects in deal perceptions. Journal of Product \& Brand Management, 16(2), 142-147.

http://doi.org/10.1108/10610420710740016

Krishnan, H. S., \& Shapiro, S. (1996). Comparing implicit and explicit memory for brand names from advertisements. Journal of Experimental Psychology: Applied, 2(2), 147-163. http://doi.org/10.1037/1076-898X.2.2.147

Kunst-Wilson, W. R., \& Zajonc, R. B. (1980). Affective discrimination of stimuli that cannot be recognized. Science, 207(4430), 557-558. http://doi.org/10.1126/science.7352271

Lang, A. (2000). The limited capacity model of mediated message processing. Journal of Communication, 50(1), 46-70.

Lee, A. Y. (2001). The mere exposure effect: An uncertainty reduction explanation revisited. Personality and Social Psychology Bulletin, 27(10), 1255-1266.

http://doi.org/10.1177/01461672012710002

Lee, A. Y. (2002). PROCESSING: prior exposure to a target enhances the ease with which consumers can process the target in subsequent encounters, and in turn, this fluency leads to more favorable attitudes toward the target. Journal of Consumer Research, 39(4), 440-454.

Lee, A. Y., \& Aaker, J. L. (2004). Bringing the frame into focus: the influence of regulatory fit on processing fluency and persuasion. Journal of Personality and Social Psychology, 86(2), 205-18. http://doi.org/10.1037/0022-3514.86.2.205

Lee, A. Y., \& Labroo, A. A. (2004). The Effect of Conceptual and Perceptual Fluency on Brand Evaluation. Journal of Marketing Research, 41(2), 151-165.

http://doi.org/10.1509/jmkr.41.2.151.28665
Lee, J., Lin, L., \& Robertson, T. (2012). The impact of media multitasking on learning. Learning, Media and Technology, 37(March 2015), 94-104. http://doi.org/10.1080/17439884.2010.537664

Macdonald, E. K., \& Sharp, B. M. (1996). Management Perceptions of the Importance of Brand Awareness as an Indication of Advertising Effectiveness. Marketing Research On-Line, 1-15.

Macdonald, E. K., \& Sharp, B. M. (2000). Brand Awareness Effecs on Consumer Decision Making for a Common, Repeat Purchase Product: A Replication. Journal of Business Research, 48(1), 5-15. http://doi.org/10.1016/S01482963(98)00070-8

Mackey, T. B., Freeman, C., \& Oil, S. (1998). As Time Goes By: From the Industrial Revolutions to the Information Revolu- tion, by Chris Freeman and Francisco Louca . New York : Oxford University. Academy of Management Review, 306-312.

Maslow, A. H. (1937). The influence of familiarization on preference. Journal of Experimental Psychology, 21(2007), 162-180. http://doi.org/10.1037/h0053692

McCullough, J. L., \& Ostrom, T. M. (1974). Repetition of highly similar messages and attitude change. Journal of Applied Psychology, 59 (June)(3), 395-397. http://doi.org/10.1037/h0036658

McGlone, M. S., \& Tofighbakhsh, J. (2000). Birds of a feather flock conjointly (?): rhyme as reason in aphorisms. Psychological Science, 11(5), 424-8. http://doi.org/10.1111/1467-9280.00282

Mishra, H., Mishra, A., \& Nayakankuppam, D. (2006). Money: A Bias for the Whole. Journal of Consumer Research, 32(4), 541-549. http://doi.org/10.1086/500484

McGuire, W. (1985). Attitudes and attitude change In W. McGuire, G. Lindzey, \& E. Aronson (Eds.), Handbook of social psychology: Special fields and applications (Vol. II) (pp. 233-346). Hillsdale: Lawrence Erlbaum.

Monin, B. (2003). The warm glow heuristic: when liking leads to familiarity. Journal of Personality and Social Psychology, 85(6), 1035-48. http://doi.org/10.1037/0022-3514.85.6.1035 
Nedungadi, P. (1990). Recall and Consumer Consideration Sets: Influencing Choice without Altering Brand Evaluations. Journal of Consumer Research, 17(3).

Nordhielm, C. L. (2002). The Influence of Level of Processing on Advertising Repetition Effects. JOURNAL OF CONSUMER RESEARCH, 29(29), 371-382. http://doi.org/10.1086/344428

Novemsky, N., Dhar, R., Schwarz, N., \& Simonson, T. (2007). Preference fluency in choice. Journal of Marketing Research, 44(3), 347-356. http://doi.org/10.1509/jmkr.44.3.347

Oppenheimer, D. M. (2006). Consequences of erudite vernacular utilized irrespective of necessity: problems with using long words needlessly. Applied Cognitive Psychology, 20(2), 139-156. http://doi.org/10.1002/acp.1178

Oppenheimer, D. M. (2008). The secret life of fluency. Trends in Cognitive Sciences, 12(6), 237241. http://doi.org/10.1016/j.tics.2008.02.014

Pancer, E., \& Poole, M. (2016). The popularity and virality of political social media: hashtags, mentions, and links predict likes and retweets of 2016 U.S. presidential nominees' tweets. Social Influence, 4510(December), 0. http://doi.org/10.1080/15534510.2016.1265582

Peretz, I., Gaudreau, D., \& Bonnel, A.-M. (1998). Exposure effects on music preference and recognition. Memory \& Cognition, 26(5), 884-902. http://doi.org/10.3758/BF03201171

Petty, R., Cacioppo, J., \& Schumann, D. (1983). Central to The and Peripheral Role of Routes Involvement Advertising Moderating Effectiveness :. Journal of Consumer Research, 10(2), 135-146.

Pilotta, J. J., Schultz, D. E., Drenik, G., \& Rist, P. (2004). Simultaneous media usage: A critical consumer orientation to media planning. Journal of Consumer Behaviour, 3, 285-292. http://doi.org/Article

Porter, R. H., \& Winberg, J. (1999). Unique salience of maternal breast odors for newborn infants. Neuroscience and Biobehavioral Reviews. http://doi.org/10.1016/S0149-7634(98)00044-X

Reber, R., \& Schwarz, N. (1999). Effects of Perceptual Fluency on Judgments of Truth. Consciousness and Cognition, 8(3), 338-342. http://doi.org/10.1006/ccog.1999.0386
Reber, R., Schwarz, N., \& Winkielman, P. (2004). Processing fluency and aesthetic pleasure: is beauty in the perceiver's processing experience? Personality and Social Psychology Review, 8(4), 364-82. http://doi.org/10.1207/s15327957pspr0804_3

Reber, R., Winkielman, P., \& Schwarz, N. (1998). Effects of Perceptual Fluency on Affective Judgments. Psychological Science, 9(1), 45-48.

Rideout, V. J., Foehr, U. G., \& Roberts, D. F. (2010). Generation M2: Media in the Lives of 8 to 18 Year-Olds. http://doi.org/P0-4461797991366925520306

Riebe, E., \& Dawes, J. (2006). Recall of radio advertising in low and high advertising clutter formats. International Journal of Advertising, 25(1), 71-86.

Sanbonmatsu, D. M., Strayer, D. L., MedeirosWard, N., \& Watson, J. M. (2013). Who MultiTasks and Why? Multi-Tasking Ability, Perceived Multi-Tasking Ability, Impulsivity, and Sensation Seeking. PLoS ONE, 8(1). http://doi.org/10.1371/journal.pone.0054402

Schwarz, N. (2004). Metacognitive Experiences in Consumer Judgment and Decision Making. Journal of Consumer Psychology, 14(4), 332-348. http://doi.org/10.1207/s15327663jcp1404_2

Seamon, J. G., Williams, P. C., Crowley, M. J., Kim, I. J., Langer, S. A., Orne, P. J., \& Wishengrad, D. L. (1995). The mere exposure effect is based on implicit memory: Effects of stimulus type, encoding conditions, and number of exposures on recognition and affect judgments. Journal of Experimental Psychology: Learning, Memory, and Cognition, 21(3), 711-721. http://doi.org/10.1037/0278-7393.21.3.711

Shapiro, S. (1999). When an ad's influence is beyond our conscious control: Perceptual and conceptual fluency effects caused by incidental ad exposure. Journal of Consumer Research, 26(1), 16-36.

Shapiro, S., \& Nielsen, J. H. (2013). What the blind eye sees: Incidental change detection as a source of perceptual fluency. Journal of Consumer Research, 39(6), 1202-1218. http://doi.org/10.1086/667852

Smith, E. R. (2000). Subjective experience of familiarity: Functional basis in connectionist memory. In H. Bless \& J. P. Forgas (Eds.), The message within: The role of subjective experience 
in social cognition and behavior (pp. 109-124). Filadelfia: Psychology Press.

Sohn, S. (2017). Consumer processing of mobile online stores: Sources and effects of processing fluency. Journal of Retailing and Consumer Services, 36(September 2016), 137-147. http://doi.org/10.1016/j.jretconser.2017.01.008

Stang, D. J. (1974). Intuition as artifact in mere exposure studies. Journal of Personality and Social Psychology, 30(5), 647-653.

Tanner, R. J., \& Maeng, A. (2012). A Tiger and a President: Imperceptible Celebrity Facial Cues Influence Trust and Preference. Journal of Consumer Research, 39(4), 769-783. http://doi.org/10.1086/665412

Tsai, C. I., \& McGill, A. L. (2011). No Pain, No Gain? How Fluency and Construal Level Affect Consumer Confidence. Journal of Consumer Research, 37(5), 807-821. http://doi.org/10.1086/655855

Tulving, E., \& Schacter, D. L. (1990). Priming and human memory systems. Science, 247(4940).

Tversky, A., \& Kahneman, D. (1973). Availability: A heuristic for judging frequency and probability. Cognitive Psychology, 5(2), 207-232. http://doi.org/10.1016/0010-0285(73)90033-9

van Rompay, T. J. L., de Vries, P. W., \& van Venrooij, X. G. (2010). More than Words: On the Importance of Picture-Text Congruence in the Online Environment. Journal of Interactive Marketing, 24(1), 22-30. http://doi.org/10.1016/j.intmar.2009.10.003

Voorveld, H. A. M. (2011). Media multitasking and the effectiveness of combining online and radio advertising. Computers in Human Behavior, 27(6), 2200-2206.

http://doi.org/10.1016/j.chb.2011.06.016
Voorveld, H. A. M., \& Goot, M. van der. (2013). Age Differences in Media Multitasking: A Diary Study. Journal of Broadcasting \& Electronic Media, 57(3), 392-408.

http://doi.org/10.1080/08838151.2013.816709

Wajcman, J. (2008). Life in the fast lane? Towards a sociology of technology and time. British Journal of Sociology, 59(1), 59-77.

http://doi.org/10.1111/j.1468-4446.2007.00182.x

Westbrook, R. A. (1980). Intrapersonal affective influences on consumer satisfaction with products. Journal of Consumer Research, 7(1), 49. http://doi.org/10.1086/208792

Whittlesea, B. W. A. (1993). Illusions of familiarity. Journal of Experimental Psychology: Learning, Memory, and Cognition, 19(6), 12351253. http://doi.org/10.1037/0278-7393.19.6.1235

Whittlesea, B. W. A., \& Williams, L. D. (2000). The source of feelings of familiarity: the discrepancy-attribution hypothesis. Journal of Experimental Psychology. Learning, Memory, and Cognition, 26(3), 547-65.

Winkielman, P., Berridge, K. C., \& Wilbarger, J. L. (2005). Unconscious affective reactions to masked happy versus angry faces influence consumption behavior and judgments of value. Personality \& Social Psychology Bulletin, 31(1), 121-35. http://doi.org/10.1177/0146167204271309

Winkielman, P., Schwarz, N., Fazendeiro, T., \& Reber, R. (2003). The hedonic marking of processing fluency: Implications for evaluative judgment. In J. Musch \& K. C. Klauer (Eds.), The Psychology of Evaluation: Affective Processes in Cognition and Emotion (pp. 189-217). Mahwah (EUA): Lawrence Erlbaum.

Zajonc, R. B. (1968). Attitudinal effects of mere exposure. Journal of Personality and Social Psychology Monograph Supplement, 9(2), 1-27. 
A Persuasão Inconsciente: Um Modelo Conceitual sobre os Efeitos da Multitarefa de Mídia sobre a Fluência de Processamento do Consumidor

i "Processing fluency" foi traduzido de forma literal pelos autores deste artigo, visto que não se encontrou literatura sobre o assunto publicados em língua portuguesa até a data de publicação deste artigo.

ii Os autores informam que a fluência de recuperação (recovery fluency - tradução livre do inglês para o português realizada pelos autores do presente trabalho), importante no julgamento e tomada de decisão por estar ligada à facilidade com que os indivíduos se lembram ou recuperam informações relevantes de forma espontânea (Novemsky, Dhar, Schwarz, \& Simonson, 2007), não será objeto de estudo do presente artigo, uma vez que seus efeitos são semelhantes aos da fluência de processamento no comportamento do consumidor de uma forma geral (Chang, 2013; Tsai \& McGill, 2011; Winkielman et al., 2003)

iii Perceptual fluency foi traduzido de forma literal pelos autores deste artigo, visto que não se encontrou literatura sobre o assunto publicado em língua portuguesa até a data de publicação deste artigo.

iv Conceptual fluency foi traduzido de forma literal pelos autores deste artigo, visto que não se encontrou literatura sobre o assunto publicados em língua portuguesa até a data de publicação deste artigo.

${ }^{v}$ Termo traduzido de forma literal pelos autores deste artigo, visto que não se encontrou na literatura em língua portuguesa até a data de publicação deste artigo.

vi Termo traduzido de forma literal pelos autores deste artigo, visto que não se encontrou na literatura em língua portuguesa até a data de publicação deste artigo.

vii Termo traduzido de forma literal pelos autores deste artigo, visto que não se encontrou na literatura em língua portuguesa até a data de publicação deste artigo.

viii Termo traduzido de forma literal pelos autores deste artigo, visto que não se encontrou na literatura em língua portuguesa até a data de publicação deste artigo.

${ }^{\text {ix }}$ Media multitasking foi traduzido de forma literal pelos autores deste artigo, visto que não se encontrou literatura sobre o assunto publicado em língua portuguesa até a data de publicação deste artigo. 